\title{
Clinical Significance of Atypical Squamous Cells of Undetermined Significance among Patients Undergoing Cervical Conization
}

\author{
Mai Nishimura ${ }^{1}$, Takashi Miyatake ${ }^{1 *}$, Ayaka Nakashima ${ }^{1}$, Ai Miyoshi $^{1}$, Mayuko \\ Mimura $^{1}$, Masaaki Nagamatsu ${ }^{1}$, Kazuhide Ogita ${ }^{2}$, Takeshi Yokoi ${ }^{1}$
}

\begin{abstract}
Background: Atypical squamous cells of undetermined significance (ASCUS) feature a wide variety of cervical cells, including benign and malignant examples. The management of ASCUS is complicated. Guidelines for office gynecology in Japan recommend performing a high-risk human papillomavirus (HPV) test as a rule. The guidelines also recommend repeat cervical cytology after 6 and 12 months, or immediate colposcopy. The purpose of this study was to determine the clinical significance of ASCUS. Materials and Methods: Between January 2012 and December 2014, a total of 162 patients underwent cervical conization for cervical intraepithelial neoplasia grade 3 (CIN3), carcinoma in situ, squamous cell carcinoma, microinvasive squamous cell carcinoma, and adenocarcinoma in situ at our hospital. The results of cervical cytology prior to conization, the pathology after conization, and high-risk HPV testing were obtained from clinical records and analyzed retrospectively. Results: Based on cervical cytology, $31(19.1 \%)$ of 162 patients were primarily diagnosed with ASCUS. Among these, $25(80.6 \%)$ were positive for high-risk HPV, and the test results of the remaining 6 patients $(19.4 \%)$ were uncertain. In the final pathological diagnosis after conization, $27(87.1 \%)$ and 4 patients $(12.9 \%)$ were diagnosed with CIN3 and carcinoma in situ, respectively. Conclusions: Although ASCUS is known as a low-risk abnormal cervical cytology, approximately $20 \%$ of patients who underwent cervical conization had ASCUS. The relationship between the cervical cytology of ASCUS and the final pathological results for CIN3 or invasive carcinoma should be investigated statistically. In cases of ASCUS, we recommend HPV tests or colposcopic examination rather than cytological follow-up, because of the risk of missing CIN3 or more advanced disease.
\end{abstract}

Keywords: ASC-US - colposcopy - abnormal cervical cytology

Asian Pac J Cancer Prev, 16 (18), 8145-8147

\section{Introduction}

Cervical cytology has become a standard screening test for cervical cancer. In addition, the human papillomavirus (HPV) test and colposcopic examination have improved the detection of cervical neoplasia and cervical cancer. Atypical squamous cells of undetermined significance (ASC-US) are considered low-risk abnormal cervical cytology.

However, ASC-US contain a wide variety of cervical cells including benign and malignant cells. An important point in the management of ASC-US is risk stratification. Testing for high-risk HPV subtypes is the preferred method. Guidelines for office gynecology in Japan (2014) also recommend repeat cervical cytology after 6 and 12 months, or immediate colposcopy. The purpose of this study was to determine which strategy is better considering the cost-effectiveness and sensitivity for premalignant lesion detection.

\section{Materials and Methods}

In order to determine relative accuracy of diagnosis, we here retrospectively analyzed the findings of cervical cytology, pathology after conization, and results of highrisk HPV testing of patients who underwent cervical conization for cervical intraepithelial neoplasia grade 3 (CIN3), carcinoma in situ (CIS), squamous cell carcinoma (SCC), microinvasive SCC, or adenocarcinoma in situ, at Kaizuka City Hospital, Japan, between January 2012 and December 2014.

We also analyzed the results of high-risk HPV tests for all examinees who were diagnosed with atypical squamous cells of undetermined significance (ASC-US) for the first time in the same period. Those patients who were diagnosed negative for intraepithelial lesion or malignancy (NILM) and those who had previously undergone punch biopsy or cervical conization were excluded from the analysis. 


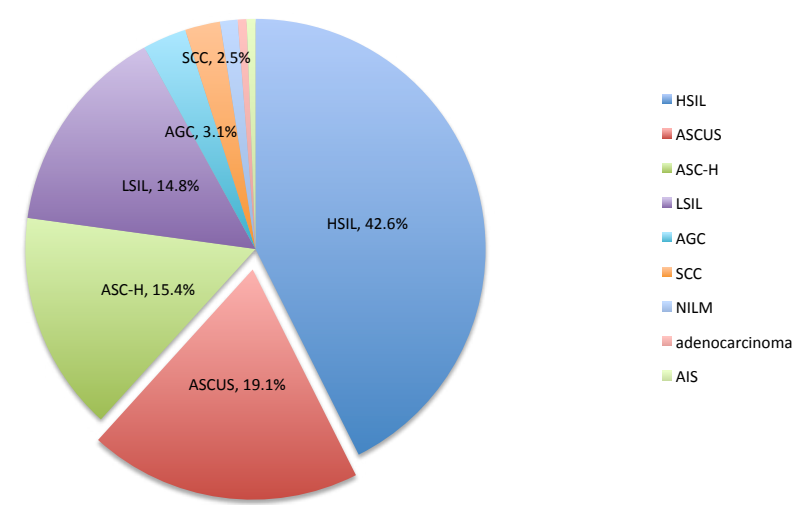

Figure 1. Cervical Cytology of Women who were Underwent Cervical Conization

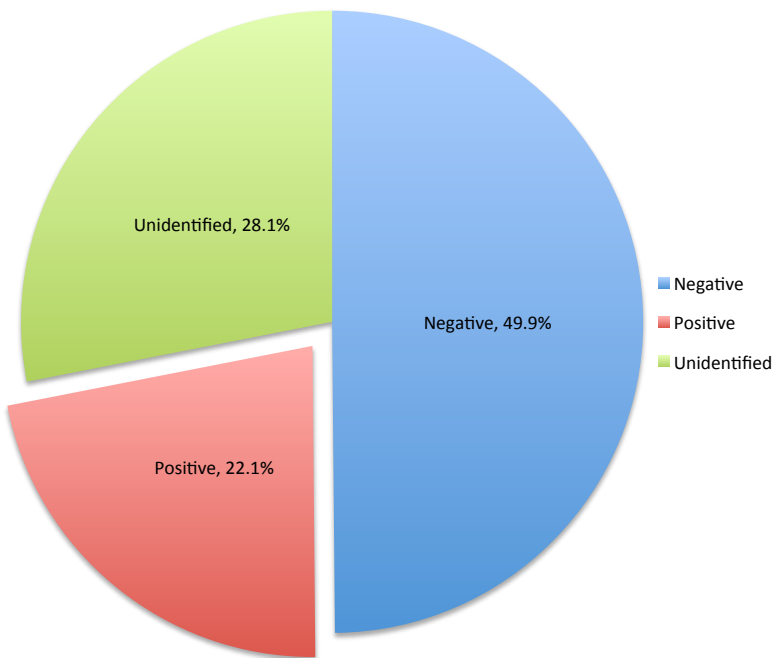

Figure 2. High-risk HPV Test Results of Patients who were Diagnosed ASC-US for the First Time

\section{Results}

A total of 162 women underwent cervical conization. The median patient age was 36 years, and the range was 19-77 years. Cervical cytology results were as follows: high-grade squamous intraepithelial lesions, 69 (42.6\%) cases; ASC-US, 31 (19.1\%) cases; atypical squamous cells where high-grade squamous intraepithelial lesions cannot be excluded, 25 (15.4\%) cases; low-grade intraepithelial lesions (LSIL), 24 (14.8\%) cases; atypical glandular cells, 5 (3.1\%) cases; SCC, 4 (2.5\%) cases; NILM, 2 (1.2\%) cases; adenocarcinoma, $1(0.6 \%)$ case; and adenocarcinoma in situ, $1(0.6 \%)$ case (Figure. 1). Among 31 women with ASC-US, 29 (93.5\%) were diagnosed with CIN3 using colposcopic biopsies, and $2(6.5 \%)$ were diagnosed with CIS. Of the 31 patients, $25(80.6 \%)$ were positive for high-risk HPV and the HPV test was not performed for $6(19.4 \%)$ patients (HPV uncertain). After conization, 27 women $(87.1 \%)$ were finally diagnosed with CIN3 and 4 women (12.9\%) were diagnosed with CIS.

In total, 825 women were diagnosed with ASC-US in the same period and 349 patients were diagnosed with ASC-US for the first time. The median patient age $(n=349)$ was 43 years, and the range was 14-90 years. Among 349 patients (Figure 2), 22 (6.3\%) underwent conization, 174 (49.9\%) were negative for high-risk HPV, 77 (22.0\%) were positive for high-risk HPV, and HPV tests were not performed in $98(28.1 \%)$ patients. Among patients with ASC-US, $30.7 \%$ (77 of 251 [77 + 174]) were positive for high-risk HPV.

\section{Discussion}

ASC-US is considered a low-risk abnormal cytology result. High-risk HPV tests are used for risk stratification. In cervical cytology alone, ASC-US accounts for $6.9 \%$ of CIN2, $2.6 \%$ of CIN3, and $0.18 \%$ of cervical cancer cases (Katki HA et al., 2013). Among ASC-US patients who tested positive for high-risk HPV, CIN2 was diagnosed in $18 \%, \mathrm{CIN} 3$ in $6.8 \%$, and cervical cancer in $0.41 \%$ of patients. However, in patients who tested negative for high-risk HPV, CIN2 was diagnosed in $1.1 \%$ and CIN3 in $0.43 \%$ of patients (Katki et al., 2013).

Three strategies for further evaluation of ASCUS, which include immediate colposcopy, HPV triage (colposcopy only if HPV-positive), or repeat cytology every 6 months, were investigated in the ASC-US/LSIL triage study (ASCUS-LSIL Triage Study (ALTS) Group, 2003). The sensitivity of CIN3 detection was the highest in the HPV triage group $(72.3 \%)$, while the sensitivity was similar in the immediate colposcopy group (53.6\%) and repeat cytology group (54.6\%). Although the ASCUS/LSIL triage study was performed before the 2001 Bethesda system was introduced, subsequent studies revealed similar findings (Arbyn et al., 2004; Arbyn et al., 2005; Li et al., 2011; Arbyn et al., 2013). Therefore, it is generally accepted that high-risk HPV testing was more sensitive than other strategies. However, in some countries where high-risk HPV testing is not a routine practice, alternative approaches to evaluation of ASC-US are needed. Many investigators recommend immediate colposcopy rather than repeat cytology (Mojgan et al., 2011; Thitichaya et al., 2015; Yenrudee et at., 2015). Thitichaya et al. (2015) retrospectively studied the risk factors of persisted abnormal Pap smear in patients with ASC-US. High risk factors for persistent ASC-US or more in subsequent test were premenopausal status, HIV infected patients and non-oral contraceptive pills users, so they concluded that immediate colposcopy should be offered for women who had these risk factors (Thitichaya et al., 2015). Similarly, Yenrudee et al. (2015). patients with ASC-US who had 3 or more sexual partners, low education and low income were at an increased risk of CIN 2-3 and recommend immediate colposcopy for early detection of high-grade CIN (Yenrudee et at., 2015). Although, further investigation of risk factors for highgrade CIN would be necessary, patients with ASC-US who had risk factors may be candidates for immediate colposcopy (Mojgan et al., 2011; Tokmak et al., 2014; Thitichaya et al., 2015 ; Yenrudee et at., 2015).

In terms of cost-effectiveness, the high-risk HPV test was considered less expensive than repeat cytology in the United States (Kim et al., 2002; Kulasingam et al., 2006; Legood et al., 2006). However, these cost analyses were influenced by the population setting in the study. In the United Kingdom, the trial of management of borderline and other low grade abnormal smears 
(TOMBOLA) group investigated the cost effectiveness of repeat cytology (cytological surveillance), colposcopy for biopsy and recall of necessary lesions (biopsy and recall), and colposcopy with immediate treatment based on colposcopic appearance (immediate treatment) (TOMBOLA Group, 2009). Of the three approaches, cytological surveillance was the most inexpensive; biopsy and recall was associated with the most average qualityadjusted life years per person, while immediate treatment offered the lowest costs per case of cervical intraepithelial neoplasia. The TOMBOLA group concluded that there is no compelling reason to favor a particular follow-up method over the others. In another study setting, authors from Turkey reported that high-risk HPV testing followed by colposcopy is more costly than immediate colposcopy (Mehmet et al., 2013).

In the present study, $6.3 \%$ (22 of 349) of patients with ASC-US had an indication (CIN3 or greater malignant disease) of conization, and $30.7 \%$ were positive for highrisk HPV. The ratios of CIN3 and CIS from ASC-US cytology are relatively higher than those in the literature (Katki et al., 2013). Guidelines for office gynecology in Japan and the American Society for Colposcopy and Cervical Pathology guidelines for cervical cytology recommend colposcopy in cases of a positive high-risk HPV test. In brief, according to our study, 50 (22 HPVpositive and $28 \mathrm{HPV}$-unknown) of 100 patients with ASC-US were referred for colposcopy and 6 patients were finally diagnosed with CIN3 or CIS. The remaining $50 \mathrm{HPV}$-negative patients are excluded from colposcopic examination. In Japan, the costs of high-risk HPV testing, cervical cytology, and colposcopy are $\$ 49, \$ 26$, and $\$ 62.5$, respectively. For the detection of 6 patients from 100 ASC-US cases where $30 \%$ of cases are HPV-positive, the total costs are estimated to be $\$ 6,775$ for HPV triage and $\$ 6,250$ for immediate colposcopy. The cost of repeat cytology is high and depends on the sensitivity of cytology and the required number of colposcopic examinations. It is hypothesized that the immediate colposcopy is the most inexpensive approach in Japan, however, as for the costeffectiveness in Japan, further prospective investigation of ASC-US cases would be necessary.

We prioritize high-risk HPV testing because of its sensitivity. However, if high-risk HPV testing is not available, we recommend immediate colposcopic examination rather than repeat cytology, because, to date, it is not clear which of these management approaches is more cost-effective, and the proportion of positive high-risk HPV tests is relatively high. For patients, repeat cytology is time-consuming because of the longer follow-up period. Although a randomized controlled trial including several centers is needed to prove our conclusion, the strength of our study is that it is the first study to explain the relatively high proportion of conization in patients with ASC-US and to describe the advantage of immediate colposcopy.

\section{Acknowledgements}

No funds were provided for this study. There are no conflicts of interest to declare.

\section{References}

Arbyn M, Buntinx F, Van Ranst M, et al (2004). Virologic versus cytologic triage of women with equivocal Pap smears: a meta-analysis of the accuracy to detect high-grade intraepithelial neoplasia. J Natl Cancer Inst, 96, 280-93.

Arbyn M, Paraskevaidis E, Martin-Hirsch P, Prendiville W, Dillner J (2005). Clinical utility of HPV-DNA detection: triage of minor cervical lesions, follow-up of women treated for high-grade CIN: an update of pooled evidence. Gynecol Oncol, 99, 7-11.

Arbyn M, Roelens J, Simoens C et al (2013). Human papillomavirus testing versus repeat cytology for triage of minor cytological cervical lesions. Cochrane Database Syst $\operatorname{Rev}, \mathbf{3}, 8054$.

ASCUS-LSIL Triage Study (ALTS) Group (2003). Results of a randomized trial on the management of cytology interpretations of atypical squamous cells of undetermined significance. Am J Obstet Gynecol, 188, 1383-92.

Jiang L, Zeng Yan, Li J et al (2011). Performance of high-risk human papillomavirus testing in the triage of abnormal cervical cytology among Chinese younger women in Shanghai, China. Asian Pac J Cnacer Prev, 12, 2963-68.

Katki HA, Schiffman M, Castle PE, et al (2013). Benchmarking CIN 3+ risk as the basis for incorporating HPV and Pap cotesting into cervical screening and management guidelines. J Low Genit Tract Dis, 17, 28-35.

Katki HA, Schiffman M, Castle PE, et al (2013). Five-year risks of CIN 3+ and cervical cancer among women with HPV testing of ASC-US Pap results. J Low Genit Tract Dis, 17, 36-42.

Kececioglu M, Seckin B, Baser E, et al (2013). Cost and effectiveness comparison of immediate colposcopy versus human papillomavirus DNA testing in management of atypical squamous cells of undetermined significance in Turkish women. Asian Pac J Cancer Prev, 14, 511-14.

Kim JJ, Wright TC, Goldie SJ (2002). Cost-effectiveness of alternative triage strategies for atypical squamous cells of undetermined significance. JAMA, 287, 2382-90.

Kulasingam SL, Kim JJ, Lawrence WF, et al (2006). Costeffectiveness analysis based on the atypical squamous cells of undetermined significance/low-grade squamous intraepithelial lesion Triage Study (ALTS). J Natl Cancer Inst, 98, 92-100.

Legood R, Gray A, Wolstenholme J, Moss S (2006). Lifetime effects, costs, and cost effectiveness of testing for human papillomavirus to manage low grade cytological abnormalities: results of the NHS pilot studies. BMJ, 332, 79-85.

Mojgan KZ, Fariba Binesh, Zohre Kazemi, et al (2011). Value of colposcopy in the early diagnosis of cervical cancer in patients with abnormal Pap smears at Shahid Sadoughi Hospital, Yazd. Asian Pac J Cancer Prev, 12, 3439-41.

Tokmak A, Guzel AI, Ozgu E, et al (2014). Clinical significance of atypical squamous cells of undetermined significance in detecting preinvasive cervical lesions in post-menopausal Turkish women. Asian Pac J Cancer Prev, 15, 6639-41.

Thitichaya P, Panya S, Watcharin C, Kornkarn B, Komsun S (2015). Colposcopy requirement of Papanicolaou smear after atypical squamous cells of undetermined significance (ASC-US) by follow-up protocol in an urban gynaecology clinic, a retrospective study in Thailand. Asian Pac J Cancer Prev, 16, 4977-80.

TOMBOLA Group (2009). Cytological surveillance compared with immediate referral for colposcopy in management of women with low grade cervical abnormalities: multicentre randomised controlled trial. $B M J, 339,2546-56$. 